\title{
Antibody-Based In Vivo PET Imaging Detects Amyloid- $\beta$ Reduction in Alzheimer Transgenic Mice After BACE-1 Inhibition
}

\author{
Silvio R. Meier ${ }^{1}$, Stina Syvänen ${ }^{1}$, Greta Hultqvist ${ }^{2}$, Xiaotian T. Fang ${ }^{1}$, Sahar Roshanbin ${ }^{1}$, Lars Lannfelt ${ }^{1,3}$, Ulf Neumann ${ }^{4}$, \\ and Dag Sehlin ${ }^{1}$ \\ ${ }^{I}$ Department of Public Health and Caring Sciences/Geriatrics, Uppsala University, Uppsala, Sweden; ${ }^{2}$ Department of \\ Pharmaceutical Biosciences, Uppsala University, Uppsala, Sweden; ${ }^{3}$ BioArctic AB, Stockholm, Sweden; and ${ }^{4}$ Neuroscience Research, \\ Novartis Institutes for BioMedical Research, Basel, Switzerland
}

Visualization of amyloid- $\beta(A \beta)$ pathology with PET has become an important tool for making a specific clinical diagnosis of Alzheimer disease (AD). However, the available amyloid PET radioligands, such as ${ }^{11} \mathrm{C}$-Pittsburgh compound $B$, reflect levels of insoluble $A \beta$ plaques but do not capture soluble and protofibrillar $A \beta$ forms. Furthermore, the plaque load appears to be fairly static during clinical stages of $A D$ and may not be affected by $A \beta$-reducing treatments. The aim of the present study was to investigate whether a novel PET radioligand based on an antibody directed toward soluble aggregates of $A \beta$ can be used to detect changes in $A \beta$ levels during disease progression and after treatment with a $\beta$-secretase (BACE-1) inhibitor. Methods: One set of transgenic mice (tg-ArcSwe, a model of $A \beta$ pathology) aged between 7 and 16 mo underwent PET with the $A \beta$ protofibril-selective radioligand ${ }^{124} \mathrm{I}-\mathrm{RmAb} 158-\mathrm{scFv} 8 \mathrm{D} 3$ (where RmAb is recombinant mouse monoclonal antibody and scFv is single-chain variable fragment) to follow progression of $A \beta$ pathology in the brain. $A$ second set of tg-ArcSwe mice, aged $10 \mathrm{mo}$, were treated with the BACE-1 inhibitor NB-360 for 3 mo and compared with an untreated control group. A third set of tg-ArcSwe mice, also aged $10 \mathrm{mo}$, underwent PET as a baseline group. Brain tissue was isolated after PET to determine levels of $A \beta$ by ELISA and immunohistochemistry. Results: The concentration of ${ }^{124}$ I-RmAb158-scFv8D3, as measured in vivo with $P E T$, increased with age and corresponded well with the ex vivo autoradiography and $A \beta$ immunohistochemistry results. Mice treated with NB-360 showed significantly lower in vivo PET signals than untreated animals and were similar to the baseline animals. The decreased ${ }^{124}$ I-RmAb158-ScFv8D3 concentrations in NB-360-treated mice, as quantified with $\mathrm{PET}$, corresponded well with the decreased $A \beta$ levels measured in postmortem brain. Conclusion: Several treatments for $A D$ are in phase 2 and 3 clinical trials, but the possibility of studying treatment effects in vivo on the important, nonfibrillar, forms of $A \beta$ is limited. This study demonstrated the ability of the $A \beta$ protofibril-selective radioligand ${ }^{124}{ }^{1}-\mathrm{RmAb} 158$-scFv8D3 to follow disease progression and detect treatment effects with PET imaging in tg-ArcSwe mice.

Received Apr. 17, 2018; revision accepted May 7, 2018.

For correspondence or reprints contact: Dag Sehlin, Department of Public Health and Caring Sciences/Geriatrics, Uppsala University, Dag Hammarskjölds väg 20, 75185 Uppsala, Sweden.

E-mail: dag.sehlin@pubcare.uu.se

Published online May 31, 2018.

Immediate Open Access: Creative Commons Attribution 4.0 International License (CC BY) allows users to share and adapt with attribution, excluding materials credited to previous publications. License: https://creativecommons. org/licenses/by/4.0/. Details: http://jnm.snmjournals.org/site/misc/permission. xhtml.

COPYRIGHT @ 2018 by the Society of Nuclear Medicine and Molecular Imaging.
Key Words: Alzheimer's disease; positron emission tomography (PET); antibody-based radioligand; BACE-1 inhibitor NB-360; amyloid- $\beta$

J Nucl Med 2018; 59:1885-1891

DOI: 10.2967/jnumed.118.213140

A lzheimer disease (AD) is the most common neurodegenerative disease, and the aging of the population will increase the number of people affected. However, no treatment is yet available to halt the pathologic changes underlying disease progression. The amyloid hypothesis (1) states that aggregation of the amyloid- $\beta$ $(\mathrm{A} \beta)$ protein eventually leads to neurodegeneration and finally to dementia. To reduce $A \beta$ pathology, immunotherapy studies have been conducted on several $A \beta$ antibodies (2). Aducanumab, which targets $A \beta$ aggregates, recently showed benefits in $A D$ patients (3), and BAN2401 (4), the humanized version of mAb158 $(5,6)$, which targets soluble $A \beta$ protofibrils, is in a phase $2 b$ clinical trial. In addition, several low-molecular-weight $\beta$-secretase (BACE-1) inhibitors, aimed at reducing $A \beta$ production, are in clinical trials.

Because the pathologic $A \beta$ accumulation occurs over many years, there is a crucial need for sensitive, specific, and representative diagnostic tools to follow disease progression and the effects of disease-modifying treatments in clinical studies.

Several small-molecule PET ligands, such as ${ }^{11} \mathrm{C}-$ Pittsburgh compound $\mathrm{B}(\mathrm{PiB})$ and ${ }^{18} \mathrm{~F}$-florbetaben, visualize amyloid plaques and have become important tools in $\mathrm{AD}$ diagnostics. However, amyloid PET may be positive years before any clinical symptoms appear (7) and shows an early saturation during disease progression (8). In addition, diagnosis with ${ }^{11} \mathrm{C}-\mathrm{PiB}$ and analogs has limited value when the $\mathrm{A} \beta$ pathology is diffuse, because diffuse plaques largely lack the $\beta$-sheet fibrillar structure to which these radioligands bind (9). Studies on $\mathrm{A} \beta$ toxicity have indicated that soluble aggregates, such as oligomers and protofibrils, are the most neurotoxic $A \beta$ species (10-12). In addition, the brain level of soluble $A \beta$ protofibrils seems to be a more dynamic indicator of disease severity and may thus be a better marker for disease progression than insoluble plaques $(8,11)$. It is also likely that novel treatments directed at decreasing $A \beta$ production ( $\beta$-secretase inhibitors) or enhancing $A \beta$ clearance (immunotherapy) will reduce levels of soluble $A \beta$ before an effect on plaque load can be detected. Treatment effects could thus be difficult to monitor with 
the current amyloid PET tracers. In fact, a recent study found only subtle changes in amyloid with the PET tracer ${ }^{18} \mathrm{~F}$-florbetapir in APPPS1-21 mice treated with a BACE-1 inhibitor (JNJ-49146981) for 12 mo (13). Hence, a PET radioligand that visualizes forms other than insoluble, fibrillar $A \beta$ could be an important tool for detecting drug effects in clinical trials.

We previously described the radioligand ${ }^{124} \mathrm{I}-\mathrm{RmAb} 158-\mathrm{scFv} 8 \mathrm{D} 3$ (where $\mathrm{RmAb}$ is recombinant mouse monoclonal antibody and $\mathrm{scFv}$ is single-chain variable fragment), which is based on mAb158, that binds selectively to soluble $A \beta$ protofibrils, with moderate and low cross-reactivity to $\mathrm{A} \beta$ fibrils and monomers, respectively $(6,14,15)$. Antibodies, because of their large molecular size, are generally characterized by limited passage across the blood-brain barrier. To enhance brain uptake of mAb158 and enable its use as a PET radioligand, it was functionalized with 2 single-chain variable fragments of the transferrin receptor antibody 8D3 (16). This conjugation leads to active transcytosis via the transferrin receptor into the brain (17). Several similar constructs $(15,18,19)$ have been used to image the progressive accumulation of soluble $A \beta$ aggregates in transgenic mice harboring the Arctic (A $\beta$ precursor protein $[A \beta P P] A \beta E 693 G)$ and the Swedish (A $\beta P P$ KM670/671NL) $A \beta P P$ mutations (tg-ArcSwe mice (20)) at different ages, demonstrating the potential of bispecific antibodies as neuro-PET radioligands.

The aim of the present study was, in a first step, to investigate the ability of ${ }^{124} \mathrm{I}-\mathrm{RmAb} 158-\mathrm{scFv} 8 \mathrm{D} 3$ to follow disease progression, that is, escalating brain $A \beta$ pathology, in the tg-ArcSwe mouse model. To achieve this aim, in vivo ${ }^{124} \mathrm{I}-\mathrm{RmAb} 158$-scFv8D3 PET in $\operatorname{tg}$-ArcSwe mice of different ages was compared with ex vivo measurement of brain radioactivity and $A \beta$ pathology in postmortem analyzed brain tissue from the same mice.

In a second set of mice, $A \beta$ reduction after treatment with the BACE-1 inhibitor NB-360 (21) was studied to investigate the ability of ${ }^{124} \mathrm{I}-\mathrm{RmAb} 158$-scFv8D3 to image the reverse effect, that is, a decrease in brain A $\beta$ pathology. In previous studies, NB-360 has shown a robust $A \beta$ reduction and a promising pharmacokinetic profile in AßPP transgenic mice. In addition, NB-360 has demonstrated a beneficial effect on cellular, long-range circuitry and memory in APP23xPS45 transgenic mice (22). Thus, in tg-ArcSwe mice we conducted a preclinical NB-360 treatment study, designed to resemble a clinical study of a new drug candidate, using ${ }^{124} \mathrm{I}-\mathrm{RmAb} 158-$ scFv8D3 PET imaging to quantify the treatment effect in vivo.

\section{MATERIALS AND METHODS}

\section{Animals}

tg-ArcSwe mice (20) were scanned with PET to study disease progression at the age of 7, 10, 13, and 16 mo. The effect of NB-360 treatment was investigated in mice aged $10 \mathrm{mo}$ at the start of the treatment, that is, at an age characterized by moderate A $\beta$ pathology (23) but no detectable ${ }^{11} \mathrm{C}$-PiB PET signal (15). The number of mice in each age group is displayed in Supplemental Table 1 (supplemental materials are available at http://jnm.snmjournals.org).

Throughout the experiment, the animals were kept with free access to food and water in rooms with controlled temperature and humidity in an animal facility at Uppsala University. All experiments were approved by the Uppsala County Animal Ethics board (approval C17/14) following the rules and regulations of the Swedish Animal Welfare Agency and complied with the European Communities Council Directive of September 22, 2010.

\section{Radioligand}

The recently described radioligand ${ }^{124} \mathrm{I}-\mathrm{RmAb} 158-\mathrm{scF}$ F8D3 was used for antibody-based PET imaging. Its expression and pharmacokinetics were described by Hultqvist et al. in 2017 (17). The radioligand is based on the well-studied antibody mAb158, which displays a selective binding to $\mathrm{A} \beta$ protofibrils $(6,14,15)$. To increase the transport of $\mathrm{mAb} 158$ across the blood-brain barrier into the brain, 2 single-chain variable fragments of the transferrin receptor antibody 8D3 (16) were attached using short linkers to the $\mathrm{C}$ termini of the light chains of $\mathrm{mAb} 158$. This step enables monovalent transferrin receptor binding, which leads to efficient transcytosis over the blood-brain barrier. RmAb158-scFv8D3 was recombinantly expressed in Expi293 cells and purified as described previously (17).

\section{Radiochemistry}

RmAb158-scFv8D3 was labeled with ${ }^{124}$ I using direct radioiodination (24). Radiolabeling was done in 6 batches; a $119 \pm 31.3 \mathrm{MBq}{ }^{124} \mathrm{I}$ solution (PerkinElmer Inc.) was preincubated for $15 \mathrm{~min}$ with $\mathrm{NaI}$ at a concentration of $10 \mu \mathrm{M}$. RmAb158-scFv8D3 was mixed with phosphatebuffered saline (PBS) and added to the iodine solution at $0.6 \mu \mathrm{g} / \mathrm{MBq}$ to a final volume of $380 \mu \mathrm{L}$. The reaction was activated by addition of $40 \mu \mathrm{L}$ of chloramine- $\mathrm{T}$ solution $(1 \mathrm{mg} / \mathrm{mL})$ and quenched after $120 \mathrm{~s}$ by addition of $80 \mu \mathrm{L}$ of sodium metabisulfite solution $(1 \mathrm{mg} / \mathrm{mL})$. The radiolabeled protein was purified of free iodine and low-molecularweight components with a disposable NAP-5 size-exclusion column with a molecular-weight cutoff of $5 \mathrm{kDa}$ (GE Healthcare). The highmolecular-weight fraction containing the labeled protein was eluted with $1 \mathrm{~mL}$ of PBS.

\section{PET Imaging}

PET imaging was performed with ${ }^{124} \mathrm{I}-\mathrm{RmAb} 158-\mathrm{scFv} 8 \mathrm{D} 3 \mathrm{using}$ a Triumph Trimodality System (TriFoil Imaging, Inc.). To reduce thyroidal uptake of ${ }^{124} \mathrm{I}$, the mice were given $0.5 \% \mathrm{NaI}$ in the drinking water $1 \mathrm{~d}$ before radioligand injection and then $0.2 \% \mathrm{NaI}$ until the PET scan. The mice in the disease-progression investigation were intravenously injected with $14.8 \pm 2.6 \mathrm{MBq}$ of ${ }^{124} \mathrm{I}-\mathrm{RmAb} 158$ $\mathrm{scFv} 8 \mathrm{D} 3$ with a specific activity of $234 \mathrm{MBq} / \mathrm{nmol}$ and scanned $4 \mathrm{~d}$ afterward. The mice in the NB-360 treatment experiment were intravenously injected with $7.4 \pm 1.3 \mathrm{MBq}$ of ${ }^{124} \mathrm{I}-\mathrm{RmAb} 158-\mathrm{scFv} 8 \mathrm{D} 3$ with a specific activity of $161 \pm 12.7 \mathrm{MBq} / \mathrm{nmol}$ and scanned $6 \mathrm{~d}$ afterward. Before undergoing PET, the animals were anesthetized with $2.5 \%$ isoflurane, moved to the heated scanner bed, and kept anesthetized with $1.5 \%-2 \%$ isoflurane during the scan. The duration of the scan was $60 \mathrm{~min}$, followed by a CT examination of $3 \mathrm{~min}$ (field of view, $8.0 \mathrm{~cm}$ ).

Directly after scanning, the mice underwent a 2-min intracardiac perfusion with $0.9 \% \mathrm{NaCl}$. Brain and blood samples were collected, and ex vivo radioactivity was measured with a well counter (GE Healthcare)

PET data were reconstructed using 3-dimensional ordered-subsets expectation maximization (20 iterations). CT raw files were reconstructed using filtered backprojection. All subsequent processing of the PET and CT images was performed using the imaging software Amide, version 1.0.4 (25). The CT scans were manually aligned with a T2-weighted, MRI-based mouse brain atlas (26) containing outlined regions of interest for whole brain, cortex, and hippocampus. The PET images were then aligned with the CT images, and the atlas and PET data could be quantified in regions of interest.

\section{Ex Vivo Autoradiography}

Frozen right hemispheres of ${ }^{125} \mathrm{I}-\mathrm{RmAb} 158$-scFv8D3-injected mice were cryosectioned $(20 \mu \mathrm{m})$ with a Leica $\mathrm{CM} 1850$ at $-25^{\circ} \mathrm{C}$. Sections were stored at $-20^{\circ} \mathrm{C}$ until use. Fresh tissue sections were placed in an X-ray cassette and exposed to positron-sensitive phosphor screens (MultiSensitive; PerkinElmer) for $6 \mathrm{~d}$. The plates were then scanned with a Cyclone Plus Imager system (Perkin Elmer) at a resolution of 600 dpi. Images were analyzed by ImageJ software, version 1.51 . 


\section{NB-360 Treatment}

Three groups of mice were investigated (Fig. 1; Supplemental Table 1). A NB-360-treated group was provided food containing the BACE-1 inhibitor NB-360 (Novartis) at a concentration of $0.5 \mathrm{~g} / \mathrm{kg}$ for $3 \mathrm{mo}$, whereas a vehicle group was maintained on control food until the age of 13 mo. Mice from 4 litters were randomly allocated to the NB-360- or vehicle-treated groups, and all mice were studied during the same period. A baseline group, included to provide an estimate of the $A \beta$ pathology level before treatment, had free access to control food until the age of $10 \mathrm{mo}$, when PET scans and analyses were conducted. All groups were scanned on the same occasion.

\section{Brain Sample Preparation}

After perfusion, brains were removed and dissected in half. The right hemisphere was either fixed in 4\% PFA and embedded in paraffin or frozen on dry ice for later autoradiography analysis. The left hemisphere was immediately frozen on dry ice for further extraction and measurement of $A \beta$ concentrations, as previously described $(15,26)$. In short, brain tissue was homogenized with a tissue grinder (Teflon [The Chemours Co.] pestle), $2 \times 10$ strokes on ice, at a 1:5 weight-to-volume ratio in Tris-buffered saline (TBS) with cOmplete Protease Inhibitor Cocktail Tablets (Roche). A $250-\mu \mathrm{L}$ volume of the homogenate was mixed with another $250 \mu \mathrm{L}$ of TBS and centrifuged for $1 \mathrm{~h}$ at $16,000 \mathrm{~g}$. The supernatants were stored at $-80^{\circ} \mathrm{C}$ until analysis. For extraction of TBS insoluble proteins, $27 \mu \mathrm{L}$ of the original extract and $73 \mu \mathrm{L}$ of $96 \%$ formic acid (FA) for an FA concentration of $70 \%$ were mixed for $30 \mathrm{~s}$ with a Kimble Pestle pellet grinder (SigmaAldrich) followed by $1 \mathrm{~h}$ of centrifugation at $16,000 \mathrm{~g}$. Supernatants were stored at $-80^{\circ} \mathrm{C}$ until analysis.

\section{Biochemical A $\beta$ Analyses}

To break $A \beta$ aggregates for better detection (27), TBS extracts from all transgenic mice were supplemented with $1 \%$ sodium dodecyl sulfate and kept for $5 \mathrm{~min}$ at $95^{\circ} \mathrm{C}$ in a compact dry heat block (ThermoFisher) and then further diluted to $0.05 \%$ sodium dodecyl sulfate.

Prepared samples were analyzed with a V-PLEX A $\beta$ Peptide Panel 1 Kit (Meso Scale Diagnostics) for $A \beta 38, A \beta 40$, and $A \beta 42$ using neoepitope-specific antibodies to the different $A \beta C$ termini in combination with $6 \mathrm{E} 10$, which binds near the $\mathrm{A} \beta \mathrm{N}$ terminus. The assay was conducted according to the user's manual, and the plates were read using a Meso Scale Diagnostics imager.

$A \beta$ protofibrils and oligomers were measured with a homogeneous ELISA, using the A $\beta \mathrm{N}$ terminus-specific 82E1 (IBL International/ Tecan Trading AG) as both capture and detection antibody and a calibration curve of synthetic $\mathrm{A} \beta$ protofibrils for quantification (18). A 96-well half-area plate was coated overnight with $12.5 \mathrm{ng}$ of $82 \mathrm{E} 1$ per well, followed by blocking with $1 \%$ bovine serum albumin in PBS.

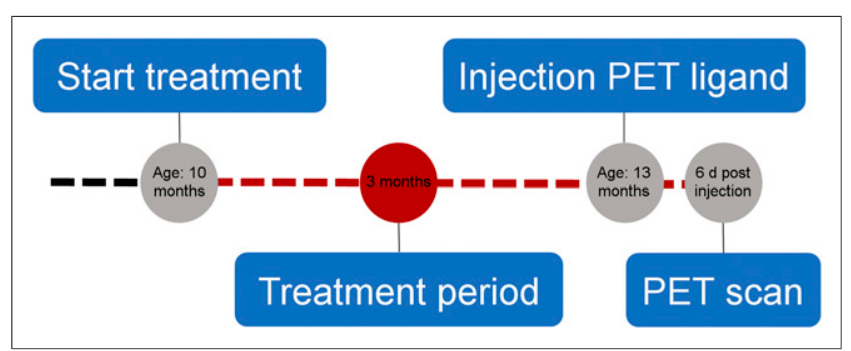

FIGURE 1. Overview of treatment and PET imaging. At age of $10 \mathrm{mo}$, animals were given either food containing BACE-1 inhibitor NB-360 or control food. At age of $13 \mathrm{mo}$, animals were injected with radioligand and were scanned with PET $6 \mathrm{~d}$ later. A third group was scanned with PET and analyzed at baseline age of 10 mo for comparison at starting point.
TBS brain extracts were diluted 1:25 and incubated overnight at $4^{\circ} \mathrm{C}$. Soluble $A \beta$ aggregates with a size of at least a dimer were then detected with biotinylated $82 \mathrm{E} 1(0.25 \mu \mathrm{g} / \mathrm{mL})$ and streptavidin-horseradish peroxidase (Mabtech $\mathrm{AB}$ ), diluted 1:4,000. Signals were developed with $\mathrm{K}$ blue aqueous tetramethylbenzidine substrate (Neogen Corp.) and read with a spectrophotometer at $450 \mathrm{~nm}$.

For ELISA measurement of total $A \beta x-40$ and $A \beta x-42,96-w e l l$ plates were coated overnight with $100 \mathrm{ng}$ per well of polyclonal rabbit anti-A $\beta 40$ or anti-A $\beta 42$ (Agrisera) and blocked with $1 \%$ bovine serum albumin in PBS. FA-soluble brain extracts were neutralized with $2 \mathrm{M}$ Tris and diluted $1: 10,000$ for $\mathrm{A} \beta 40$ and 1:750 for $\mathrm{A} \beta 42$ analysis and then were incubated overnight at $4{ }^{\circ} \mathrm{C}$. After incubation with biotinylated 6E10 (Nordic BioSite) $(0.5 \mu \mathrm{g} / \mathrm{mL})$ and streptavidin-horseradish peroxidase (Mabtech $\mathrm{AB}$ ), diluted 1:5,000, signals were developed and read as above. All sample and secondary antibody dilutions were made in ELISA incubation buffer $(0.1 \%$ bovine serum albumin, $0.05 \%$ polysorbate 20 in PBS).

\section{Immunohistochemistry}

Five-micrometer sections from fixed, paraffin-embedded right-brain hemispheres were processed with an electronic rotary microtome (Hm340E; Thermofisher). Sections were deparaffinized and incubated in $3 \% \mathrm{H}_{2} \mathrm{O}_{2}$ and $10 \%$ methanol in water for $15 \mathrm{~min}$. Nonspecific binding was blocked with $3 \%$ bovine serum albumin in PBS-polysorbate 20 $(0.1 \%)$ for $1 \mathrm{~h}$ and incubated overnight with a polyclonal rabbit-antiA 340 antibody (Agrisera). Sections were incubated with biotinylated goat anti-rabbit antibody (Vector Laboratories Inc.) for $2 \mathrm{~h}$ at room temperature, followed by PBS washes and a 45-min incubation with avidin/biotin complex (Vector Laboratories). The staining was visualized by 3-min 3,3'-diaminobenzidine development and mounted with DPX mounting medium (a mixture of distyrene, tricresyl phosphate and xylene; SigmaAldrich). Pictures were captured with a DXM1200F microscope (Nikon Instruments Inc.) at a magnification of $\times 5$ and further processed with Photoshop CC photomerge (Adobe) to a whole-brain panorama.

For $A \beta$ and glial fibrillary acidic protein (GFAP) immunofluorescence double staining, sections were deparaffinized, underwent antigen retrieval in citrate buffer at $86^{\circ} \mathrm{C}$ for $20 \mathrm{~min}$, and were further permeabilized and blocked for $1 \mathrm{~h}$ with $5 \%$ normal goat serum and $0.3 \%$ Triton X-100 (The Dow Chemical Co.) in PBS. A $\beta$ and GFAP were stained with 6E10 (Nordic BioSite) and anti-GFAP (Dako), respectively. Alexa Fluor 488 anti-mouse and Alexa Fluor 555 anti-rabbit (Life Technologies) were used as secondary antibodies. Pictures were captured with an LSM700 confocal laser scanning microscope (Zeiss), and the software Zen 2012 was used for image processing.

\section{Statistics}

All statistical analyses and graphs were done in Prism, version 6 (GraphPad Software, Inc.). Group results are reported as mean \pm SD. Data were analyzed with 1-way ANOVA followed by the Bonferroni post hoc test.

\section{RESULTS}

$\operatorname{tg}$-ArcSwe mice between 7 and 16 mo old were scanned using ${ }^{124}$ I-RmAb158-scFv8D3 PET to study the ability of the radioligand to visualize progression of $A \beta$ brain pathology. PET images corresponded well with ex vivo autoradiography images of brain sections prepared from perfused brain-that is, tissue devoid of any background signal from blood (Fig. 2). A $\beta 40$ staining displayed a clear increase in $A \beta$ burden with age. Further, the overlay of the autoradiography and the $A \beta 40$ staining showed a high degree of colocalization between areas with high $\mathrm{A} \beta 40$ pathology and high retention of ${ }^{124} \mathrm{I}-\mathrm{RmAb} 158-\mathrm{scFv} 8 \mathrm{D} 3$ (Fig. 2). 


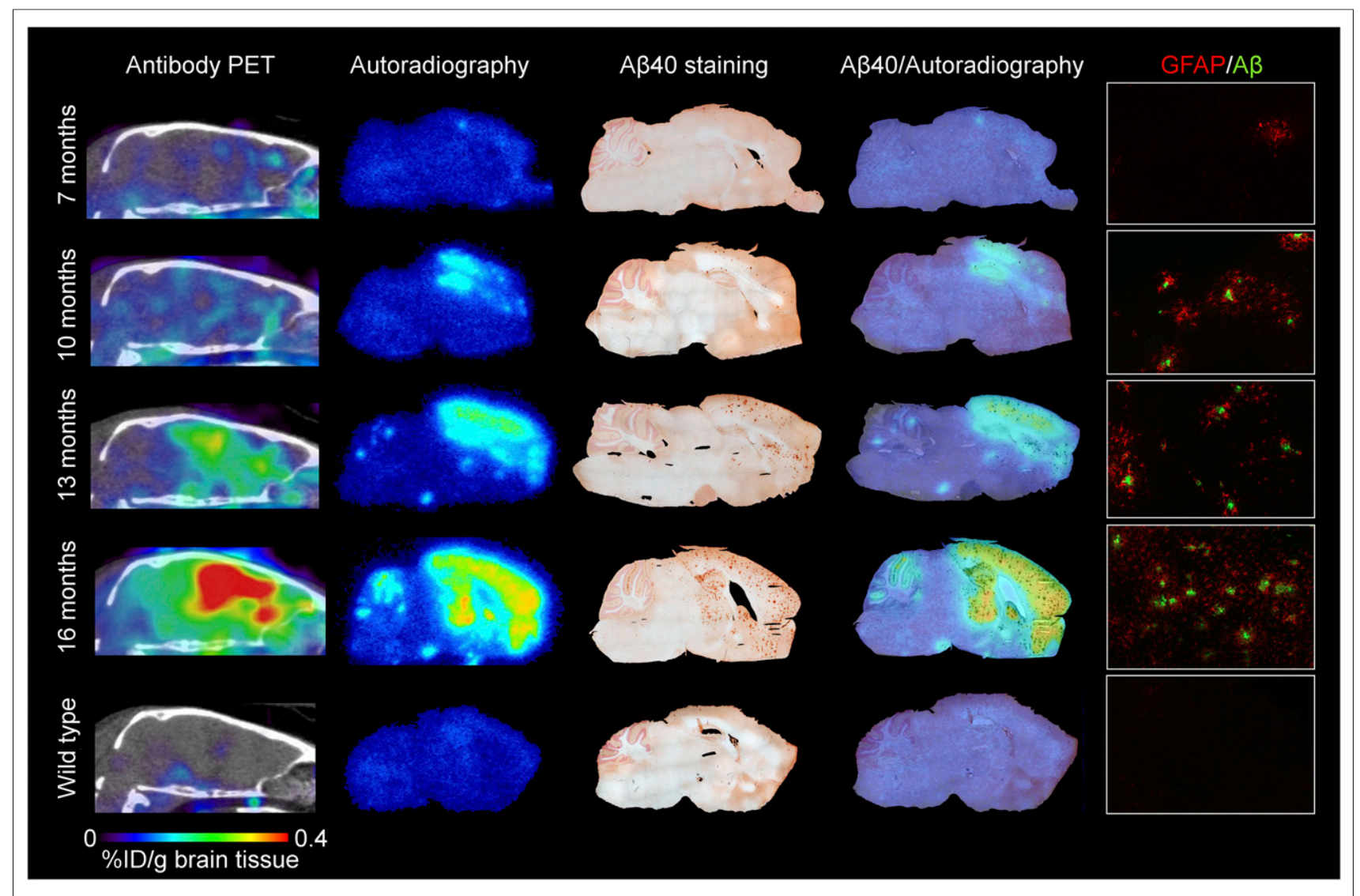

FIGURE 2. Disease progression and comparison between PET and ex vivo analysis. Representative images show disease progression in tgArcSwe mice from age of 7 mo up to 16 mo. From left to right, in vivo PET images with ${ }^{124}$ I-RmAb158-scFv8D3 are compared with corresponding ex vivo autoradiography and $A \beta 40$ staining of same individual. Overlay of $A \beta 40$ staining and ex vivo autoradiography highlights colocalization of injected ${ }^{124}$ I-RmAb158-scFv8D3 and A 40 pathology. GFAP/A $\beta$ column shows activated astrocytes around $A \beta$ deposits at $\times 20$ magnification in cortex. \%ID = percentage injected dose.

At the age of $7 \mathrm{mo}$, astrocyte activation measured with GFAP immunofluorescence was rare and limited to certain spots in the cortex. With increasing plaque load at the ages of 10 and 13 mo, astrocytes were activated around plaques. At the age of $16 \mathrm{mo}$, a comprehensive, areawide activation could be observed (Fig. 2).

In the second set of mice, PET scans were obtained after 3 mo of treatment with NB-360-containing food pellets or control food. A group of younger mice, representing the baseline, was also studied. ${ }^{124} \mathrm{I}-\mathrm{RmAb} 158-\mathrm{scFv} 8 \mathrm{D} 3$ brain retention in the baseline group and the NB-360-treated group was low, and there was no notable difference between these groups, indicating that progression of $\mathrm{A} \beta$ pathology was halted by BACE-1 inhibitor treatment. In contrast, the group that had received control food showed a high PET signal in areas of abundant $A \beta$ pathology (Fig. 3).

In line with the in vivo results, whole-brain $A \beta$ staining revealed a higher $A \beta$ burden in the vehicle group than in the NB-360 or baseline group. Pathology was observed mainly in the cortex, the hippocampus, and, with further disease progression in the vehicle group, the thalamus (Fig. 3). A $\beta /$ GFAP double staining of the prefrontal cortex showed higher GFAP activity in the vehicle group than in the NB-360 or baseline group. Higher activation could especially be observed around plaques (Fig. 3).

Image-based quantification of radioactivity showed that there was no significant difference in any of the studied regions between the baseline and NB-360-treated groups, whereas the vehicle group showed significantly higher activity in all brain regions, most pronounced in the cortex and hippocampus (Fig. 4; $P=$ 0.002 in whole brain, $P<0.0001$ in cortex, and $P<0.0001$ in hippocampus). To verify the PET results, $A \beta$ levels were biochemically assessed with an electrochemiluminescence immunoassay (MSD Technology Platform; Meso Scale Diagnostics) and ELISA in TBS-soluble and -insoluble brain extracts from the same mice that had undergone PET. There was no significant difference in TBS-soluble A $\beta 38, A \beta 40$, or A $\beta 42$ levels between the NB-360 and baseline groups. However, a small trend toward slightly higher levels of all studied $A \beta$ species could be observed in the NB-360 group (Figs. 5A-5C). The vehicle group showed significantly higher levels of soluble $A \beta 38, A \beta 40$, and $A \beta 42$ than the baseline or treated animals $(P=0.0001, P=0.0013$, and $P=0.0001$, respectively) (Figs. 5A-5C). Similar results were observed for soluble $A \beta$ aggregates (Fig. 5D), with untreated mice displaying significantly higher levels than treated $(P<0.0001)$ or baseline $(P<0.0001)$ mice. Here, a trend was observed toward lower levels in the NB-360 group than in the baseline group. A ratio of soluble $\mathrm{A} \beta$ aggregates over total soluble $A \beta$ displayed a nearly significant difference $(P=0.051)$ between the baseline group $(1.9 \pm 1.4)$ and the NB-360 group $(0.8 \pm 0.5)$, indicating a relative decrease in $\mathrm{A} \beta$ oligomerization as a result of BACE-1 inhibition.

ELISA analysis of FA-soluble brain extracts, which represent total $\mathrm{A} \beta 40$ and $\mathrm{A} \beta 42$, including plaques, also showed similar 


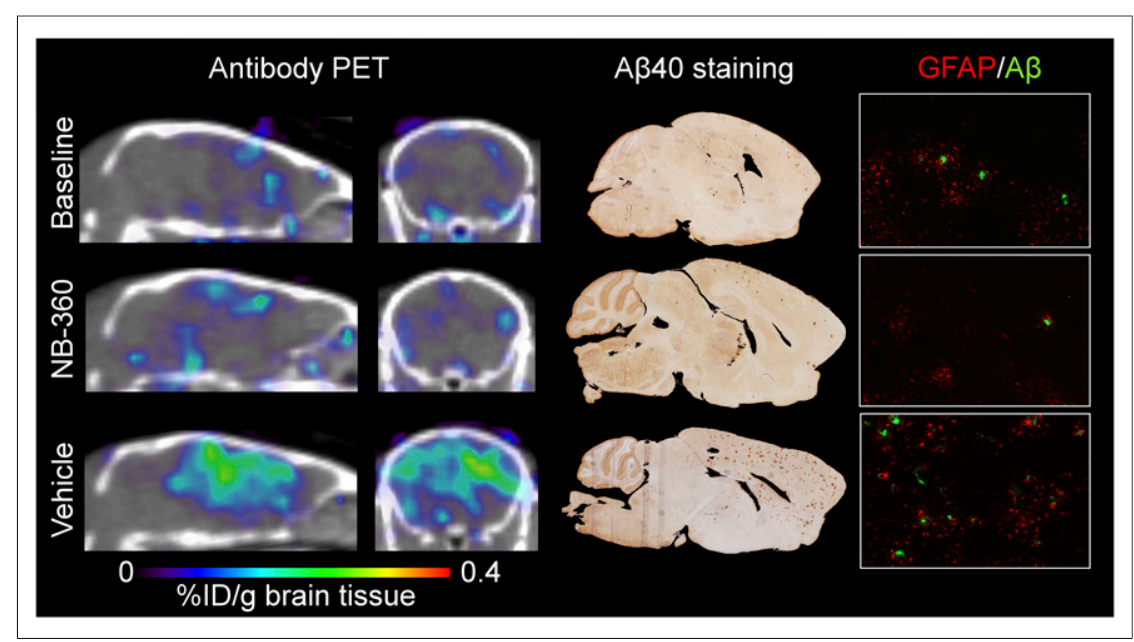

FIGURE 3. Overview of PET imaging, A $\beta$, and GFAP pathology after NB-360 treatment. Comparison is made of 3 groups of mice: baseline, NB-360, and vehicle. Sagittal and coronal PET images are at left, with brain concentrations of ${ }^{124} \mathrm{I}-\mathrm{RmAb} 158-\mathrm{scFv8D} 3$ expressed as percentage injected dose (\%ID) per gram of brain tissue. Corresponding A 340 -stained whole-brain images show total $A \beta$ burden, including plaques. $A \beta$ and GFAP staining demonstrates colocalization of $A \beta$ deposits and reactive astrocytes at $\times 20$ magnification.

results. A $\beta$ levels in baseline and NB-360-treated mice were equally low, indicating a similar plaque load in treated and baseline mice. Again, the vehicle group had significantly higher $\mathrm{A} \beta$ levels (for $\mathrm{A} \beta 40, P<0.0001$, and for $\mathrm{A} \beta 42, P<0.0001$ ) (Figs. $5 \mathrm{E}-5 \mathrm{~F})$.

\section{DISCUSSION}

During the past decade, amyloid PET imaging, such as that using ${ }^{11} \mathrm{C}-\mathrm{PiB}$, has become an important tool for reliable diagnosis of $A \beta$ plaque pathology. However, whereas amyloid plaques are a hallmark of $\mathrm{AD}$, soluble $\mathrm{A} \beta$ may be a more dynamic marker of disease progression. Moreover, because the drugs currently in development are aimed mainly at reducing soluble $\mathrm{A} \beta$, the existing amyloid PET ligands may not be optimal for quantifying the effects of treatment. In the present study, we demonstrated that a recombinantly produced PET ligand derived from the $A \beta$ protofibril-selective antibody mAb158 can be used to visualize and quantify the progression of $\mathrm{A} \beta$ pathology in transgenic tg-ArcSwe mice and, further, detects changes in brain $A \beta$ levels related to an $A \beta$-reducing treatment.

PET imaging requires a certain density of the target in the studied tissue to produce a signal that can be reliably quantified. Although brain concentrations of soluble $A \beta$ protofibrils, the target of $\mathrm{mAb} 158$, are already elevated at 2 mo of age in tg-ArcSwe mice (27), the levels are initially low until plaques start to develop at around 6 mo of age (20), catalyzing $A \beta$ aggregation and accumulation of soluble aggregates (28). With the PET ligand ${ }^{124} \mathrm{I}-\mathrm{RmAb} 158-\mathrm{scFv} 8 \mathrm{D} 3$, $A \beta$ pathology was already detected at the age of $7 \mathrm{mo}$, with a broad dynamic range and an increasing PET signal up to the age of 16 mo (Fig. 2; Supplemental Fig. 1A). PET results correlated well with ex vivo-measured radioactivity and $\mathrm{A} \beta$ pathology (Supplemental Fig. 1B; Fig. 2). This finding is to be compared with ${ }^{11} \mathrm{C}$-PiB PET imaging, which in a previous study barely detected $A \beta$ pathology at the age of 12 mo but gave detectable signals at 18 mo (15), despite the dense, AD-like nature of tgArcSwe plaques (23). ${ }^{11} \mathrm{C}-\mathrm{PiB}$, binding to the amyloid plaque core, thus seems to require a high density of plaques to produce a signal. In contrast, although dependent on the presence of plaques, ${ }^{124} \mathrm{I}-$ RmAb158-scFv8D3 seems to detect a more dynamic and abundant pool of $A \beta$, associated with the plaques. On the basis of these findings, a study was designed to investigate the sensitivity of ${ }^{124} \mathrm{I}-\mathrm{RmAb} 158-\mathrm{scFv} 8 \mathrm{D} 3 \mathrm{PET}$ to $\mathrm{A} \beta$-reducing treatment at an early stage of $A \beta$ pathology.

The reduction of $A \beta$ accumulation in $\operatorname{tg}$-ArcSwe mice was achieved with the BACE-1 inhibitor NB-360, which previously gave a prominent $A \beta$ reduction in APP51/16 mice (21). After 3 mo of treatment, PET images obtained with the A $\beta$ protofibrilselective radioligand ${ }^{124} \mathrm{I}-\mathrm{RmAb} 158-\mathrm{scFv} 8 \mathrm{D} 3$ clearly visualized markedly reduced $\mathrm{A} \beta$ pathology in NB360-treated mice in comparison with the vehicle group, similar to baseline (Fig. 3). Quantification of PET data confirmed this result, with equally low signals in baseline and NB-360-treated mice and a significantly higher signal in the vehicle group, especially in brain regions with abundant $\mathrm{A} \beta$ pathology, such as the cortex and hippocampus (Fig. 4). These results suggest that $\mathrm{A} \beta$ accumulation was essentially halted during the 3 mo of treatment and that the PET ligand clearly detected this treatment effect in vivo at a stage of pathology that is below the detection limit of ${ }^{11} \mathrm{C}$-PiB PET imaging in this animal model.

ELISA analyses of TBS brain extracts confirmed the PET results. Animals without treatment had a normal development of pathology from the age of 10 mo to the age

FIGURE 4. PET quantification of different brain regions. Image-based radioactivity is expressed as percentage injected dose (\%ID) per gram of brain tissue, for whole brain (A), cortex (B), and hippocampus (C). While there was no significant difference between baseline and NB-360, vehicle group showed significantly higher activity in all brain regions $(P=0.002$ in whole brain, $P<$ 0.0001 in cortex, and $P<0.0001$ in hippocampus). Values represent group mean with SD. ${ }^{\star \star} P<0.01$. ${ }^{\star \star *} P<0.001$. ${ }^{* \star \star} P<0.0001$. 


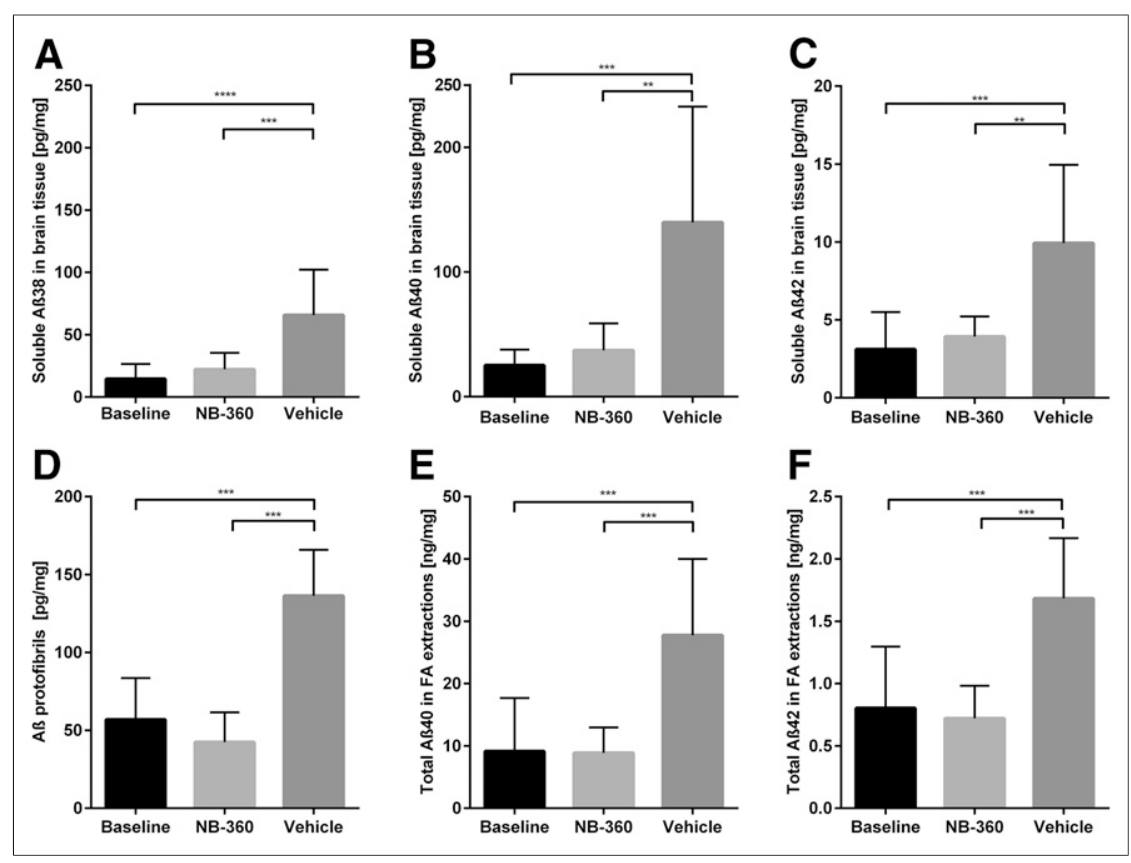

FIGURE 5. $A \beta$ analysis in postmortem brain. (A-C) TBS-soluble $A \beta 38(A), A \beta 40(B)$, and $A \beta 42$ (C) in brain extracts from mice after PET scanning. Bars show group mean and SD. Baseline $(n=$ 9) and NB-360-treated animals $(n=9)$ showed significantly lower levels of all 3 A $\beta$ species than did vehicle animals $(n=11 ; P<0.001)$. (D) Similar pattern was revealed with $A \beta$ oligomer/ protofibril-specific ELISA, showing significant difference between NB-360 and vehicle animals $(P<0.0001)$. (E and F) Total $A \beta 40(E)$ and $A \beta 42(F)$ load, measured in FA brain extracts, showed similar results. ${ }^{\star \star} P<0.01$. ${ }^{\star \star \star} P<0.001 .{ }^{\star \star \star \star} P<0.0001$. in the area surrounding amyloid plaques (28). A decreased plaque load thus implies fewer oligomerization sites and a reduction in the formation of plaque-associated, protofibrillar $A \beta$. Such an $A \beta$ reduction was previously reported after NB-360 treatment in APP23xPS45 transgenic mice (22). In the present study, ELISA analyses of postmortem brain revealed an overall reduction in soluble $A \beta$ aggregates after BACE-1 inhibition, and notably, a smaller proportion of soluble $A \beta$ seemed to be in an aggregated state. The substantially decreased in vivo PET signal observed here is therefore likely to represent this reduction in soluble $A \beta$ aggregates. Altogether, these findings highlight potential for measuring soluble $A \beta$ aggregates in vivo to monitor disease progression and detect effects of $A \beta$ reducing treatment. These findings may also explain the pronounced difference in PET signal achieved with ${ }^{124}$ I-RmAb158-scFv8D3 after BACE1 inhibition, as compared with the modest differences detected with the smallmolecule PET ligand ${ }^{18} \mathrm{~F}$-florbetapir (13), which visualizes the dense core of amyloid plaques. of $13 \mathrm{mo}$, with the usual range of variation when compared with previous investigations $(5,20,23)$. In contrast, levels of soluble $A \beta$ aggregates (Fig. 5D), the primary target for ${ }^{124} \mathrm{I}-\mathrm{RmAb} 158-\mathrm{scFv} 8 \mathrm{D} 3$, were 3 -fold lower in treated mice than in their untreated littermates and were similar to the level in baseline mice. Similarly, levels of total soluble $A \beta 38, A \beta 40$, and $A \beta 42$ were 3-fold lower in treated animals (Figs. 5A-5C), almost to the baseline level, suggesting that BACE-1 inhibition maintained low levels of soluble $A \beta$ but did not induce an overall reduction over time. Interestingly, although not statistically significant $(P=0.051)$, the relative amount of aggregated $\mathrm{A} \beta$ in the soluble pool (soluble $A \beta$ aggregates/total soluble $A \beta$ ) was lower in the treated group than in the baseline group, suggesting that the inhibited $A \beta$ production decreased the rate of aggregation.

As a consequence of reduced production and aggregation, $A \beta$ deposition and plaque formation were halted in NB-360-treated animals. $A \beta$ deposits were detected with $A \beta 40$ immunohistochemistry in the prefrontal cortex and hippocampus in the baseline group and did not change over time with NB-360 treatment. However, untreated animals with a normal course of pathology reached a significantly higher plaque load during the study (Fig. 3), as was also confirmed by ELISA quantification of total $A \beta 40$ and $A \beta 42$ in FA-soluble brain extracts (Figs. 5E-5F). The distinct GFAP staining around plaques seen in the vehicle group was reduced in treated mice, indicating a prominent effect of NB-360 treatment also on neuroinflammation.

Although the decreased plaque load per se could potentially contribute to the reduced PET signal observed here, previous studies with mAb158-derived ligands have shown little correlation between PET signal and insoluble A $\beta$. In contrast, binding was observed around plaques, and there was a good correlation with soluble $A \beta$ protofibrils (15). Soluble $A \beta$ aggregates have been reported to form

\section{CONCLUSION}

Antibody-based PET imaging of soluble $A \beta$ protofibrils is a sensitive tool for following progression of brain $A \beta$ pathology and the treatment effects achieved by inhibition of $A \beta$ production. This study is a step toward a method that might be used in future preclinical and clinical studies of novel AD drug candidates.

\section{DISCLOSURE}

Ulf Neumann is an employee and shareholder of Novartis Pharma AG, Basel, Switzerland. Lars Lannfelt is a founder and shareholder of BioArctic AB, Stockholm, Sweden. Financial support was granted from the Swedish Research Council (grant 2017-02413), Alzheimerfonden, Hjärnfonden, Torsten Söderbergs stiftelse, Hedlunds stiftelse, Stiftelsen Fondkistan, Åhlén-stiftelsen, Stiftelsen Sigurd och Elsa Goljes minne, Stohnes stiftelse, Stiftelsen för Gamla tjänarinnor, Magnus Bergwalls stiftelse, and the Uppsala Berzelii Technology Centre for Neurodiagnostics. The molecular imaging work in this study was performed at the SciLifeLab Pilot Facility for Preclinical PET-MRI, a Swedish nationally available imaging platform at Uppsala University, Sweden, financed by the Knut and Alice Wallenberg Foundation. No other potential conflict of interest relevant to this article was reported.

\section{ACKNOWLEDGMENTS}

We thank Dr. Derya Shimshek, Novartis, for supplying the NB-360 food pellets; Professor Lars Nilsson for developing the mouse model used in this study; and BioArctic AB for sharing the mAb158 sequence. 


\section{REFERENCES}

1. Hardy JA, Higgins GA. Alzheimer's disease: the amyloid cascade hypothesis. Science. 1992;256:184-185.

2. Hung S-Y, Fu W-M. Drug candidates in clinical trials for Alzheimer's disease. J Biomed Sci. 2017;24:47.

3. Sevigny $\mathrm{J}$, Chiao $\mathrm{P}$, Bussière $\mathrm{T}$, et al. The antibody aducanumab reduces $\mathrm{A} \beta$ plaques in Alzheimer's disease. Nature. 2016;537:50-56.

4. Logovinsky V, Satlin A, Lai R, et al. Safety and tolerability of BAN2401: a clinical study in Alzheimer's disease with a protofibril selective A $\beta$ antibody. Alzheimers Res Ther. 2016;8:14.

5. Lord A, Gumucio A, Englund H, et al. An amyloid-beta protofibril-selective antibody prevents amyloid formation in a mouse model of Alzheimer's disease. Neurobiol Dis. 2009;36:425-434.

6. Englund H, Sehlin D, Johansson AS, et al. Sensitive ELISA detection of amyloid- $\beta$ protofibrils in biological samples. J Neurochem. 2007;103:334-345.

7. Chételat G, La Joie R, Villain N, et al. Amyloid imaging in cognitively normal individuals, at-risk populations and preclinical Alzheimer's disease. Neuroimage Clin. 2013;2:356-365.

8. Engler H, Forsberg A, Almkvist O, et al. Two-year follow-up of amyloid deposition in patients with Alzheimer's disease. Brain. 2006;129:2856-2866.

9. Schöll M, Wall A, Thordardottir S, et al. Low PiB PET retention in presence of pathologic CSF biomarkers in Arctic APP mutation carriers. Neurology. 2012;79: 229-236.

10. Esparza TJ, Wildburger NC, Jiang H, et al. Soluble amyloid-beta aggregates from human Alzheimer's disease brains. Sci Rep. 2016;6:38187.

11. Esparza TJ, Zhao H, Cirrito JR, et al. Amyloid-beta oligomerization in Alzheimer dementia versus high-pathology controls. Ann Neurol. 2013;73:104-119.

12. Walsh DM, Klyubin I, Fadeeva JV, et al. Naturally secreted oligomers of amyloid beta protein potently inhibit hippocampal long-term potentiation in vivo. Nature. 2002;416:535-539.

13. Deleye S, Waldron AM, Verhaeghe J, et al. Evaluation of small-animal PET outcome measures to detect disease modification induced by BACE inhibition in a transgenic mouse model of Alzheimer disease. J Nucl Med. 2017;58:1977-1983.

14. Magnusson K, Sehlin D, Syvänen S, et al. Specific uptake of an amyloid- $\beta$ protofibril-binding antibody-tracer in A $\beta P P$ transgenic mouse brain. $J$ Alzheimers Dis.2013;37:29-40.

15. Sehlin D, Fang XT, Cato L, Antoni G, Lannfelt L, Syvänen S. Antibody-based PET imaging of amyloid beta in mouse models of Alzheimer's disease. Nat Commun. 2016;7:10759.
16. Kissel K, Hamm S, Schulz M, Vecchi A, Garlanda C, Engelhardt B. Immunohistochemical localization of the murine transferrin receptor (TfR) on bloodtissue barriers using a novel anti-TfR monoclonal antibody. Histochem Cell Biol. 1998;110:63-72.

17. Hultqvist G, Syvänen S, Fang XT, Lannfelt L, Sehlin D. Bivalent brain shuttle increases antibody uptake by monovalent binding to the transferrin receptor. Theranostics. 2017;7:308-318.

18. Syvänen S, Fang XT, Hultqvist G, Meier SR, Lannfelt L, Sehlin D. A bispecific Tribody PET radioligand for visualization of amyloid-beta protofibrils: a new concept for neuroimaging. Neuroimage. 2017;148:55-63.

19. Sehlin D, Fang XT, Meier SR, Jansson M, Syvänen S. Pharmacokinetics, biodistribution and brain retention of a bispecific antibody-based PET radioligand for imaging of amyloid- $\beta$. Sci Rep. 2017;7:17254.

20. Lord A, Kalimo H, Eckman C, Zhang XQ, Lannfelt L, Nilsson LN. The Arctic Alzheimer mutation facilitates early intraneuronal $\mathrm{A} \beta$ aggregation and senile plaque formation in transgenic mice. Neurobiol Aging. 2006;27:67-77.

21. Neumann U, Rueeger H, Machauer R, et al. A novel BACE inhibitor NB-360 shows a superior pharmacological profile and robust reduction of amyloid- $\beta$ and neuroinflammation in APP transgenic mice. Mol Neurodegener. 2015; $10: 44$.

22. Keskin AD, Kekuš M, Adelsberger H, et al. BACE inhibition-dependent repair of Alzheimer's pathophysiology. Proc Natl Acad Sci USA. 2017;114:86318636.

23. Philipson O, Hammarström P, Nilsson KP, et al. A highly insoluble state of Abeta similar to that of Alzheimer's disease brain is found in Arctic APP transgenic mice. Neurobiol Aging. 2009;30:1393-1405.

24. Greenwood FC, Hunter WM, Glover JS. The preparation of ${ }^{131}$ I-labelled human growth hormone of high specific radioactivity. Biochem J. 1963;89:114-123.

25. Loening AM, Gambhir SS. AMIDE: a free software tool for multimodality medical image analysis. Mol Imaging. 2003;2:131-137.

26. Ma Y, Hof PR, Grant SC, et al. A three-dimensional digital atlas database of the adult $\mathrm{C} 57 \mathrm{BL} / 6 \mathrm{~J}$ mouse brain by magnetic resonance microscopy. Neuroscience. 2005;135:1203-1215.

27. Lord A, Englund H, Söderberg L, et al. Amyloid-beta protofibril levels correlate with spatial learning in Arctic Alzheimer's disease transgenic mice. FEBS J. 2009;276: 995-1006.

28. Koffie RM, Meyer-Luehmann M, Hashimoto T, et al. Oligomeric amyloid beta associates with postsynaptic densities and correlates with excitatory synapse loss near senile plaques. Proc Natl Acad Sci USA. 2009;106:4012-4017. 